\title{
ANÁLISE DE CONTEÚDO DA PSICOLOGIA EDUCACIONAL ${ }^{1}$
}

\author{
Arrigo Leonardo Angelini \\ Samuel Pfromm Netto \\ Nelson Rosamilha
}

\begin{abstract}
A Psicologia Educacional ocupa tradicionalmente uma posição de relevo na preparação de professores - e isto reflete o reconhecimento generalizado de que é grande a contribuição que ela pode oferecer à educação em geral e à formação dos educadores em particular. Paradoxalmente, entretanto, pouco tem sido feito em nosso meio no sentido de analisar e definir o campo, os desenvolvimentos recentes, o conteúdo, os problemas e o ensino da Psicologia Educacional. A bibliografia brasileira a respeito parece limitar-se aos trabalhos de Noemy da Silveira Rudolfer (1935) sobre a história da Psicologia Educacional, posteriormente refundido e aumentado $(1938,1961)$, Lourenço Filho (1951 a, 1951 b, 1953) e Angelini (1962).
\end{abstract}

Com o objetivo de proceder a um estudo extensivo da situação atual da Psicologia Educacional - o que é, o que tem sido, como é ensinada, seus problemas e limitações e as transformações por que vem passando os autores da presente comunicação iniciaram em fins de 1962 uma série de pesquisas, que envolvem o levantamento e exame da literatura existente a respeito, a análise do conteúdo de livros de texto e de revistas especializadas, de programas e planos de ensino, assim como a determinação de novas tendências na Psicologia Educacional, entendida esta no seu sentido mais amplo, tal como a define Andreson (1949): “A Psicologia Educacional é uma disciplina a ser estudada, uma área do conhecimento, um conjunto de aplicações de leis e princípios de uma área do conhecimento a um processo social, um conjunto de técnicas e um campo de pesquisas".

Na presente comunicação, serão expostos alguns resultados da primeira etapa do referido estudo, que consistiu na análise do conteúdo de livros e revistas de Psicologia Educacional publicados durante a década de 1950 a $1959^{2}$.

\section{Aplicação da Técnica de Análise de Conteúdo à Psicologia Educacional}

Segundo a conhecida definição de Berelson, a análise de conteúdo é "uma técnica de pesquisa para a descrição objetiva, sistemática e quantitativa do conteúdo manifesto da comunicação" (Berelson, 1952, p.18). Para que seja objetiva, tal descrição exige uma definição precisa das categorias de análise, de modo a permitir que diferentes pesquisadores possam utilizálas, obtendo os mesmos resultados; para ser sistemática, é necessário que a totalidade de conteúdo relevante seja analisada com relação a todas as categorias significativas; a quantificação permite obter informações mais precisas e objetivas sobre a frequiência da ocorrência das características do conteúdo.

Empregada originalmente em pesquisas sobre o conteúdo de jornais, a técnica de análise de conteúdo tem sido largamente utilizada em investigações sobre opinião pública e propaganda e para determinar características do conteúdo de obras literárias, didáticas e científicas, em campos como a sociologia, a psicologia e a biologia.

Os primeiros estudos da natureza da Psicologia Educacional e das características, por meio da análise de conteúdo, parecem datar da década de 1920. Em 1922, Remmers e Knight examinaram cinco compêndios de uso corrente na época chegando à conclusão de que havia entre os livros "uma decidida divergência de pontos de vista, organização e conteúdo: tão grande, na

${ }^{1}$ Comunicação apresentada à $17^{a}$ Reunião Anual da Sociedade Brasileira para o Progresso da Ciência, em Belo Horizonte, $M G$ (1965).

${ }^{2}$ Este estudo contou com o financiamento do Centro Regional de Pesquisas Educacionais Prof. Queiroz Filho e a colaboração da Profa. Célia Augusta Teixeira Marques Toledo Malta, Assistente de Pesquisa do referido Centro. 
verdade, que uma projetada comparação entre os mesmos teve que ser abandonada" (p. 406). Outras investigações foram realizadas por Goodwin Watson (1926). Worcester (1927), McPhail (1938), Jones (1949), Wolfe (1947), Blair (1948, 1949), Ryans (1955), Symonds e Jensen (1955), Goodwin Watson (1956), Tyler (1956), Robert I. Watson (1959) e Angelini (1962). Entre os critérios adotados em tais pesquisas, predominaram os seguintes: porcentagem de espaço devotado a diferentes tópicos em livros de texto, autores mais citados e porcentagem de artigos relativos a pesquisas de interesse para a Psicologia Educacional.

\section{Análise de Compêndios e de Revistas de Psicologia Educacional}

A Literatura básica de Psicologia Educacional publicada em português é limitada, precária e antiquada, de modo geral. Contamos apenas com meia dúzia de compêndios redigidos por autores nacionais, preparados para alunos de escolas normais e que não cuidaram de incluir resultados de pesquisas representativas. Há, além disso, algumas traduções de livros anteriores à década de 1940 e só há pouco dois textos norte-americanos mais recentes (Ellis, 1964: Sawrey e Telford, 1964) foram vertidos para o nosso idioma.

Em virtude disso, concluiu-se que conviria concentrar a análise de livros e de revistas em publicações editadas no exterior. Mas a impossibilidade de obter compêndios e revistas exclusivamente consagrados à Psicologia Educacional e em número significativo, editados em outros países que não os Estados Unidos e a Grã-Bretanha, levou-nos a restringir mais ainda o material para análise. Esta terminou por concentrarse em: (a)compêndios de Psicologia Educacional editados nos Estados Unidos da América do Norte, durante a década de 1950 a 1959; (b) coleções completas, igualmente relativas à década de 1950 a 1959, de duas revistas exclusivamente destinadas à Psicologia Educacional: Journal of Educational Psychology, de procedência Norte-Americana, e o Britsh Journal of Educational Psychology, editada em Londres. Convém notar que tais livros e revistas figuram entre os que o Departamento de Psicologia Educacional da Faculdade de Filosofia, Ciências e Letras da Universidade de São Paulo indica para leitura aos alunos, tal como fazem outras Faculdades.
De acordo com os registros na revista Psychological Abstracts, durante a década de 1950-1959 foram publicados nos Estados Unidos cerca de 26 compêndios de Psicologia Educacional. Desses compêndios, treze, isto é, a metade do total indicado, puderam ser localizados ou adquiridos para o presente estudo. Sua análise exaustiva permitiu a verificação da distribuição de assuntos e a freqüência com que diferentes autores são citados. Para o estabelecimento das categorias de análise, várias classificações foram previamente testadas, funcionando de maneira satisfatória aquela que Blair (1948 e 1949) derivou de suas análises e que foram empregadas posteriormente por outros autores.

Tal como constataram as pesquisas relativas a livros publicados em épocas anteriores, verificou-se que, de modo geral, cada texto concede importância maior a quatro áreas: Psicologia da Aprendizagem, Psicologia do Desenvolvimento Humano, Personalidade, Ajustamento e Orientação e Testes e Medidas. Outras áreas aparecem, em média, com porcentagem igual ou menor do que cinco por cento.

Embora varie de livro para livro o número de páginas dedicado a cada uma das quatro grandes áreas, a ordem de classificação das mesmas se mantém relativamente constante. Assim, em primeiro lugar aparece a Psicologia da Aprendizagem, ocupando maior número de páginas do que as outras áreas em doze das treze obras analisadas; a Psicologia do Desenvolvimento ocupa o segundo lugar, em sete livros; em terceiro lugar figura a Psicologia da Personalidade, Ajustamento e Orientação, em nove das obras analisadas, e em quarto lugar, correspondendo a sete livros, os Testes e Medidas.

Os resultados gerais da análise do conteúdo dos compêndios figuram na tabela 1 , juntamente com as porcentagens correspondentes ao conteúdo das revistas.

Convém esclarecer que diferentes critérios foram empregados na análise de livros e na de revistas; no primeiro caso, fez-se a contagem do número de páginas, e no segundo, foi contado o número de artigos. Não se deve esquecer, também, a natureza diversa dos dois tipos de publicação. Os compêndios destinam-se ao uso de estudantes e procuram oferecer uma visão geral do campo da Psicologia Educacional; as revistas refletem a atividade de pesquisa em curso, por parte daqueles que, Psicólogos Educacionais ou não, se interessam pela investigação de problemas de aprendi- 
zagem, ensino e desenvolvimento psicológico do indivíduo. Deve-se considerar ainda, na interpretação das porcentagens relativas ao conteúdo das revistas, que existem publicações especializadas sobre Psicologia do desenvolvimento, Personalidade, Medidas Educacionais etc., para as quais são encaminhados os resultados de numerosas pesquisas. Daí, talvez, ser mínima a porcentagem de artigos sobre psicologia do desenvolvimento, por exemplo.

Além da porcentagem de espaço devotada a diferentes tópicos, outra forma de análise do conteúdo de livros é a verificação da frequiência com que os nomes de diferentes autores são citados. Trezentos e noventa e oito autores foram citados mais de três vezes, no total dos livros examinados; destes, 33 autores $(8,2 \%)$ figuram com vinte ou mais citações. Seus nomes, postos e frequência com que são citados acham-se na tabela 2 .

A simples contagem do número de vezes em que
Tabela 1. Conteúdo de livros e revistas de Psicologia Educacional (1950 - 1959).

\begin{tabular}{lcc}
\hline Conteúdo & Livros & Revistas \\
\hline Aprendizagem & $38 \%$ & $10 \%$ \\
Desenvolvimento & 19 & 4 \\
Personalidade & 14 & 18 \\
Testes, medidas & 11 & 33 \\
Outros & 18 & 35 \\
\hline
\end{tabular}

Tratamos, então, de organizar uma outra tabela, na qual a representatividade dos autores em diferentes compêndios é comparada (v. tabela 3)

Verifica-se por esta tabela que os seis primeiros colocados na tabela anterior são também os que encontram citados em maior número de compêndios: Thorndike, Terman, Lewin, Allisson Davis, Robert J. Havighurst e Gates.

Tabela 2. Autores com vinte ou mais citações em livros de Psicologia Educacional

\begin{tabular}{|c|c|c|c|}
\hline Autor & Freqüência & Autor & Freqüência \\
\hline $1^{\circ}$ Thorndike, E. L. & 172 & $13^{\circ}$ Gesell, A. & 27 \\
\hline $2^{\circ}$ Terman, L. M. & 84 & $13^{\circ}$ Goodnough, F. L. & 27 \\
\hline 3ํ Havighurst, R. J. & 64 & $13^{\circ}$ Jersild, A.T. & 27 \\
\hline 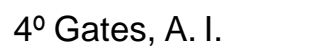 & 55 & 13oㅇson, W. C. & 27 \\
\hline $4^{\circ}$ Davis, $\mathrm{A}$ & 55 & 14º Merrill, M. A. & 25 \\
\hline $5^{\circ}$ Lewin, $\mathrm{K}$ & 52 & 15 Woodworth, R. S. & 24 \\
\hline 6oㅡㄹ Tinker, M. A & 45 & 16을. Allport, G.W. & 21 \\
\hline 7을 Pressey, S. L. & 43 & 16 Washburne, C.W. & 21 \\
\hline 8o James, W. & 33 & 16은 Witty, P. & 21 \\
\hline $9^{\circ}$ Binet, $A$. & 30 & $17^{\circ}$ Bayley, N. & 20 \\
\hline 9o Dewey, J. & 30 & 17º Brownell, W. & 20 \\
\hline 9ํ Freud, S. & 30 & $17^{\circ}$ Hollingshead, A. B. & 20 \\
\hline 9 Warner, W. L. & 30 & 17ํKearney, N. C. & 20 \\
\hline 10ํHilgard, E. R. & 29 & 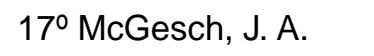 & 20 \\
\hline $11^{\circ}$ Anastasi, $\mathrm{A}$. & 28 & 17옹nggg, D. & 20 \\
\hline $11^{\circ}$ Hildreth, G. H. & 28 & $17^{\circ}$ Taba, $\mathrm{H}$ & 20 \\
\hline $12^{\circ}$ Barker, R. G. & 27 & & \\
\hline
\end{tabular}

um determinado autor é citado no total de obras analisadas pode levar à impressão de que tal autor está igualmente representado em todas as obras, o que nem sempre ocorre. Thinkor, por exemplo, que figura em sexto lugar na ordem de autores mais citados, deve tal posição ao fato de que um dos compêndios dedica grande parte do seu espaço ao exame das contribuições desse estudioso de problemas relacionados com a psicologia da leitura.
É interessante verificar como os compêndios examinados diferem quanto à variedade de autores citados. A tabela 4 apresenta os doze compêndios analisados e o número de citações que incluem em seus livros. Enquanto alguns fazem largo uso de citações de pesquisas, conceitos, teorias e pontos de vista, como é o caso de Pressey, Robinson e Herrocks, outros são relativamente reservados em suas citações, reduzindo-as a três ou quatro centenas, como Trow ou Cole e Bruco. 
Tabela 3 - Autores mais citados em livros de Psicologia Educacional, em função do número de livros nos quais são citados

\begin{tabular}{lc}
\hline Autor & № de livros \\
\hline Terman, L. M. & 11 \\
Thorndike, E. L. & 11 \\
Lewin, K. & 10 \\
Davis, A. & 8 \\
Havighurst, R. J. & 8 \\
Gates, A. I. & 7 \\
Olson, W. C. & 7 \\
Allport, G. W. & 5 \\
Barker, R. G. & 5 \\
Gesell, A. & 5 \\
James, W. & 5 \\
Jersild, A.T. & 5 \\
Jones, H. E. & 5 \\
Merril. M. A & 5 \\
Taba, H. & 5 \\
Total de Livros Examinados & 12 \\
\hline
\end{tabular}

Tabela 4 - Porcentagem do total de autores citados em cada livro de Psicologia Educacional

\begin{tabular}{lcc}
\hline Livro e data de publicação & No de autores citados & $\begin{array}{c}\text { Porcentagem do total dos } \\
\text { autores citados nos 12 livros }\end{array}$ \\
\hline Pressey, Robinson e Horrocks & 1112 & 46,1 \\
Blair, Jones e Simpson (1954) & 777 & 38,2 \\
Stephens (1951) & 566 & 23,5 \\
McDonald (1959) & 548 & 22,7 \\
Frandsen (1957) & 539 & 22,3 \\
Garrison e Gray (1955) & 485 & 20,1 \\
Commins e Fagin (1954) & 463 & 19,2 \\
Cronbach (1954) & 455 & 18,9 \\
Lindgren (1956) & 437 & 18,1 \\
Bernard (1954) & 386 & 16,0 \\
Cole e Bruce (1950) & 360 & 14,9 \\
Trow (1950) & 340 & 14,1 \\
Total de autores citados nos 12 livros & 2413 & 100,0 \\
\hline
\end{tabular}

Tendo em vista os principais desenvolvimentos sistemáticos na história recente da psicologia, cabe perguntar em que proporção estão os grandes sistemas representados nos compêndios de Psicologia Educacional. A fim de proceder a tal verificação, fez-se primeiramente uma análise dos quadros de sistemas da psicologia que constam de várias obras sobre história da Psicologia, teorias e sistemas psicológicos. De tais quadros, pareceu-nos mais satisfatório o que Marx e Hillix propõem em "Systems and theories in Psychology" (1963), ao qual foi apresentada a Psicologia Topológica de Kurt Lewin. Partindo de tal quadro, tratou-se de verificar a freqüência com que os nomes das principais figuras na formação e desenvolvimento dos grandes sistemas psicológicos são citados nos compêndios de Psicologia Educacional. A tabela 5 e a figura 1 resumem os resultados dessa verificação. 
Tabela 5 - Freqüência de citação das principais figuras na formação e desenvolvimento de sistemas psicológicos $^{3}$

\begin{tabular}{|c|c|c|c|}
\hline Sistemas e autores & № de citação por autor & Citações por sistema & $\%$ por sistema \\
\hline ASSOCIACIONISMO & & 199 & 41,4 \\
\hline Ebbinghaus & 7 & & \\
\hline Pavlov & 12 & & \\
\hline Bechterev & - & & \\
\hline Thorndike & 172 & & \\
\hline Guthrie & 8 & & \\
\hline Estes & - & & \\
\hline FUNCIONALISMO & & 110 & 22,9 \\
\hline James & 33 & & \\
\hline Dewey & 20 & & \\
\hline Angell & - & & \\
\hline Carr & - & & \\
\hline Woodworth & 24 & & \\
\hline McGeoch & 20 & & \\
\hline Melton & - & & \\
\hline Underwood & 3 & & \\
\hline BEHAVIORISMO & & 58 & 12,1 \\
\hline Meyer & - & & \\
\hline Watson & 15 & & \\
\hline Weiss & - & & \\
\hline Hunter & - & & \\
\hline Tolman & 15 & & \\
\hline Skinner & - & & \\
\hline Hull & 18 & & \\
\hline Miller & 10 & & \\
\hline Spence & - & & \\
\hline TOPOLÓGICA & & 52 & 10,8 \\
\hline Lewin & 52 & & \\
\hline PSICANÁLISE & & 44 & 9,1 \\
\hline Breuer & - & & \\
\hline Freud & 30 & & \\
\hline Adler & - & & \\
\hline Jung & 4 & & \\
\hline Rank & - & & \\
\hline Ferenczi & - & & \\
\hline Jones & - & & \\
\hline Horney & 6 & & \\
\hline Sullivan & - & & \\
\hline Fromm & 4 & & \\
\hline GESTALT & & 18 & 3,7 \\
\hline Wetheimer & 8 & & \\
\hline Koffka & 10 & & \\
\hline Kohler & - & & \\
\hline TOTAL & & & 100 \\
\hline
\end{tabular}

\footnotetext{
${ }^{3}$ Autores aparecem com um mínimo de três citações
} 
Figura 1 - Número de citações de autores ligados aos principais sistemas psicológicos, em compêndios de Psicologia Educacional

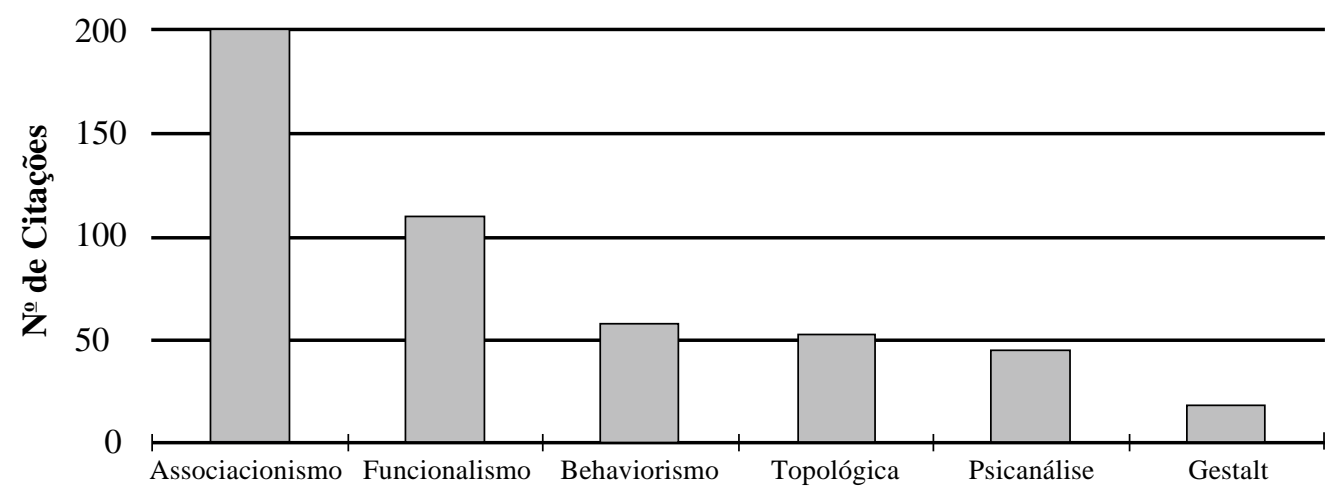

\section{Sistemas}

\section{Tipos de Estudos e Sujeitos}

Dois outros aspectos foram considerados, na parte relativa às revistas de Psicologia Educacional publicadas durante a década de 1950 a 1959: a natureza dos trabalhos publicados (tabela 6) e os tipos de sujeitos utilizados nas pesquisas relatadas (tabela 7). logo manipula deliberadamente uma variável independente; na de tipo diferencial, o psicólogo simplesmente seleciona os sujeitos de acordo com um certo critério e as variáveis (ou variável) da investigação são as medidas de tais sujeitos (Andrews, 1948, pág. 17).

Quanto aos sujeitos das pesquisas publicadas nos Estados Unidos e na Grã-Bretanha, nas revistas anali-

Tabela 6 - Natureza dos trabalhos publicados em revistas de Psicologia Educacional

\begin{tabular}{lcc}
\hline Tipo de Trabalho & \multicolumn{2}{c}{$\%$ de Artigos } \\
& Estados Unidos * & Grã-Bretanha ** \\
\hline Diferencial & 55,3 & 48,5 \\
Experimental & 22,3 & 13,1 \\
Teórico & 15,4 & 26,8 \\
Outros (“survey”, relatos, construção e validação & 7,0 & 11,6 \\
de testes etc.) & & \\
\hline
\end{tabular}

* Journal of Educational Psychology, 1950-1959

** Britsh Journal of Educational Psychology, 1950-1959

Para a determinação da natureza dos trabalhos observou-se a classificação proposta por Andrews (1948), que se refere a três tipos principais de estudos psicológicos: a experimentação, o estudo de tipo diferencial e o estudo de tipo clínico. A essa classificação acrescentou-se uma quarta categoria para permitir a inclusão de contribuições de natureza teórica. Os critérios para a definição dos diferentes tipos de estudos são os mesmos que figuram na obra de Andrews. Segundo este, na pesquisa de tipo experimental o psicó- sadas nesta investigação verificou-se que enquanto nos Estados Unidos predominam os estudantes universitários, Na Grã-Bretanha são os alunos das escolas primárias e secundárias os sujeitos mais freqüentes usados pelos pesquisadores.

\section{Resumo}

O conteúdo de treze compêndios de Psicologia Educacional, de procedência norte-americana, e de coleções das revistas "Journal of Educational Psychology" 
e "British Journal of Educational Psychology", publicados durante a década de 1950 a 1959, foi analisado sistematicamente dentro de um programa de pesquisas iniciado em 1962 e destinado à caracterização da Psicologia Educacional como área do conhecimento humano, campo de investigação científica e discipli-

\section{REFERÊNCIAS}

Anderson, G. L.(1949). Educational psychology and teacher education. Journal of Educational Psychology, 40, 275-284.

Andrews, T. G. (Ed.) (1948). Methods of psychology. New York: Wiley.

Angelini, A. L. (1962). O sentido atual da psicologia educacional. Revista de Psicologia Normal e Patológica, 1-4, 3-20.

Berelson, B. (1952). Content analysis in communication research. Glence: Free Press.

Blair, G. M. (1948). Educational psychology, its development and present status. Urbana: Bureau of Research and Service, College of Education, University of Illinois.

Blair, G. M. (1949). The content of educational psychology. Journal of Educational Psychology, 40, 267-274.

Ellis, R. S. (1949). Psicologia educacional. Trad. Haydée C. Campos. São Paulo: Nacional.

Jones, H. E. (1946). The educational psychology of persons. Journal of Educactional Psychology, 37, 513-526.

Lourenço Filho, M. B. (1951). Objeto e conteúdo da Psicologia Educacional. Anuário do Instituto de Psicologia da Universidade do Brasil, ano 1, 39-42. (a)

Lourenço Filho, M. B. (1951). A psicologia educacional e seus cursos. Anuário do Instituto de Psicologia da Universidade do Brasil, ano 1, 43-46. (b)

Lourenço Filho, M. B.(1953). Psicologia educacional. Em Klineberg, O (ed.), A psicologia moderna. Rio de Janeiro: Agir, p. 299-324. na escolar. As seguintes características principais foram consideradas na análise: (a) distribuição do conteúdo dos livros e revistas por assunto; (b) frequiência de citação de autores nos livros; (c) métodos empregados em pesquisas de Psicologia Educacional; (d) sujeitos utilizados nas pesquisas.

Mac Phail, A. H. (1938). Textbooks of introductory courses in education: an analysis of their content. Educational Administration and Supervivion, 24, 50-54.

Remmers, H. H. e Knight, F.B. (1922). The teaching of educational psychology in the United States. Journal of Educational Psychology, 13, 399-407.

Rudolfer, N. S. (1935). A evolução da psicologia educacional através de um histórico da psicologia moderna. São Paulo: Nacional.

Rudolfer, N. S. (1938). Introdução à psicologia educacional. São Paulo: Nacional, $1^{\mathrm{a}}$. ed.

Rudolfer, N. S. (1961). Introdução à psicologia educacional. São Paulo: Nacional, $2^{\text {a }}$. ed., revista e aumentada.

Ryans, D. C. (1955). Educational psychology. Annual Review of Psychology, 6, 431-454.

Sawrey, J. M. e Telford, C. W. (1964). Psicologia educacional. Trad. De Iva W. Bonow e outros. Rio de Janeiro: Ao Livros Técnico.

Symonds, P. M. e Jensen, A. R. (1955). Book reviews. Journal of Educational Psychology, 46, 56-64.

Tyler, F.T. (1956). Educational psychology. Annual Review of Psychology, 7, 283-304.

Watson, G. (1926). What shall be taught in educational psychology? Journal of Educational Psychology, 17, 577-599.

Watson, G. (1956). Educational psychology. Review of Educational Research, 26, 241-267. 
Watson, R. I. (1959). The present status of educational psychology and of educational psychologists. Evanston: Northwestern University, (mimeografado).

Wolfle, D. (1947). The sensible organization of courses in psychology. The American Psychologist,2, 437-445.
Worcester, D. A. (1927). The wide diversions of practice in first courses in educational psychology. Journal of Educational Psychology, 18, 11-17. 